\title{
EDIFICATE
}

I Congreso de Escuelas de Edificación y Arquitectura Técnica de España València, 4 y 5 de noviembre de 2021

Escuela Técnica Superior de Ingeniería de Edificación

Universitat Politècnica de València

Doi: https://doi.org/10.4995/EDIFICATE2021.2021.13540

\section{Innovación docente en el Trabajo Final de Grado de Arquitectura Técnica y Edificación. La oportunidad del Aprendizaje participativo sobre casos reales}

\section{Teaching innovation in the Final Degree Project of Technical Architecture and Building. The opportunity for participatory learning on real}

\begin{abstract}
Fernando da Casa Martín ${ }^{a}$, Antonio Baño Nieva ${ }^{b}$ y Jorge Carlos Delgado García ${ }^{c}$ aEscuela de Arquitectura,, Universidad de Alcalá. fernando.casa@uah, 'bscuela de Arquitectura, Universidad de Alcalá, antonio.banno@uah.es, 'Escuela de Arquitectura, Universidad de Alcalá, jorge.delgado@uah.es,
\end{abstract}

\begin{abstract}
The experience resulting from a Teaching Innovation Project of the University of Alcalá is presented, implementing a new teaching methodology, experienced since 2011. This subject will be endowed with the concept of a subject, in an academic year, under a collective development, with works in team, and based on the development of real cases, has allowed to achieve a high level of results in the TFG, in a limited time. It is obtained from the student who follows the process, a result that combines all the skills and abilities acquired in the degree, as well as others of a transversal nature, such as a capacity for exposing and defending their work in a professional way. The results in the case of degrees that are characterized by being professional, are even more relevant. In turn, the monitoring of the methodology has made it possible to establish a continuous Improvement Plan. Specific problems in development are analyzed both from the student's point of view and from the teacher's point of view, including cross-cutting aspects, such as those derived from the obligation of Defense before Court.
\end{abstract}

Keywords: Final Degree Project, transversality, real cases, teamwork, participatory learning, professional attributions, teaching efficiency. 


\section{Resumen}

Se presenta la experiencia fruto de un Proyecto de Innovación docente de la Universidad de Alcalá, implantando una nueva metodología docente, experimentada desde 2011. Se dota a esta materia del concepto de asignatura, en un curso académico, bajo un desarrollo colectivo, con trabajos en equipo, $y$ en base al desarrollo de caso reales. Esto ha permitido conseguir un alto nivel de resultados en el TFG, en un tiempo acotado (un curso). Se consigue del estudiante que sigue el proceso, un resultado que aúna todas las habilidades y capacidades adquiridas en la titulación, así como otras de carácter transversal, como una capacidad de exposición y defensa de su trabajo de forma profesional. Los resultados en el caso de titulaciones que están caracterizadas por ser profesionalizantes, son aún más relevantes. A su vez el seguimiento de la metodología, ha permitido establecer un continuo Plan de Mejora. Se analizan las problemáticas específicas en el desarrollo tanto desde el punto de vista del estudiante, como desde el punto de vista del profesor, incluyendo aspectos transversales, como los derivados de la obligación de Defensa ante Tribunal.

Palabras clave: Trabajo Final de Grado, transversalidad, casos reales, trabajo en equipo, aprendizaje participativo, atribuciones profesionales, eficiencia docente. 


\section{Introducción}

El Espacio Europeo de Enseñanza Superior, conocido como "plan Bolonia", se desarrolla en España, según el Real Decreto 1393/2007. En esta norma se ordenan las enseñanzas universitarias oficiales. Una novedad académica es que todas las enseñanzas oficiales de grado concluirán con la elaboración y defensa de un trabajo de fin de Grado, que ha de formar parte del plan de estudios.

Como se indica en la documentación base para el desarrollo de todas estas titulaciones (ANECA 2012), el Trabajo de Fin de Grado deberá realizarse en la fase final del plan de estudios, y estar bien diferenciado del resto de módulos o materias. Tendrá entre 6 y 30 créditos. Estará orientado a la evaluación de competencias asociadas al Título, y deberá ser defendido ante un Tribunal. Este trabajo no podrá ser reconocido o convalidado.

Este tipo de trabajos tienen un antecedente directo en los Trabajos o Proyectos Final de Carrera de las titulaciones técnicas, diferenciadas notablemente por su aspecto profesionalizante y técnico (da Casa, 2008), En otras titulaciones hay un precedente en la denominada "tesina", si bien en el resto no hay referencias previas.

La "tesina", se definía como un trabajo científico monográfico, breve y original (UNAV 2016), con una extensión que rondaba los 50-100 páginas, que era la alternativa al examen para la obtención de las Licenciaturas (UCM 2016), y que no era obligatoria, pero que daba créditos para el doctorado, por lo que en algunos modelos responde a las exigencias formales de una Tesis, pero sin el rigor de esta. Si bien se defendía públicamente lo cierto es que cada institución tenía su modalidad, que regía todos los aspectos de la misma, por lo que no es un modelo coherente como referencia.

También es de referir la necesidad de innovación en este marco. La evolución de los sistemas docentes relacionados con las enseñanzas técnicas tiene su propio paradigma. $Y$ el sector de la Edificación no es ajeno a ello. En 2021, las necesidades de un profesional, así como las oportunidades laborales, enfoques y todo lo que conlleva, han sufrido un gran cambio. Si como docentes no estamos a ello e incluso nos adelantamos a estos cambios, estaremos haciendo un flaco favor a nuestros estudiantes.

Se debe tener en cuenta una singularidad particular en el amplio espectro de otras enseñanzas de Grado, y es el carácter habilitante y la consecución, junto con el título universitario, de las correspondientes "atribuciones profesionales". Esta circunstancia obliga a que los métodos de evaluación final aplicados garanticen y comprueben que el alumno ha adquirido adecuadamente "todas" las competencias que profesionalmente se le van a exigir; es una obligación social de las Universidades. El establecimiento del trabajo fin de grado ha provocado importantes debates sobre cómo deben ser los procesos de tutorización o lo criterios de evaluación (Rullán, 2010).

Los nuevos métodos pedagógicos en el campo del aprendizaje (el Aprendizaje Activo, el Aprendizaje Cooperativo, así como el Aprendizaje Basado en Resolución de Problemas), desarrollado para materias específicas (Ojeda, 2019) (Aristizabal, 2018), aportan nuevos 
puntos de vista en cuya combinatoria puede encontrarse un proceso metodológico de aprendizaje de gran potencial en el ámbito de las Enseñanzas Técnicas.

Es a partir de esta situación en la que aparece la necesidad de buscar un modelo, y experimentarlo de modo que se conozcan las diferentes variantes y posibilidades de adaptar dicho modelo a cada titulación, más aún en la oportunidad que significa el momento con las primeras acreditaciones de los Grados que se implantaron en primer lugar.

\section{Objetivos}

El objetivo principal pues es la búsqueda de una metodología estratégica, como innovación docente, un método específico de desarrollo y evaluación de la adquisición de las competencias obtenidas por el alumno, en su proceso final de la titulación, considerando la viabilidad que el aprendizaje activo y participativo aplicado sobre casos reales.

Además, se plantean una serie de objetivos secundarios concretos, como son:

- Dotar al alumno de las habilidades y capacidades de carácter transversal, en base a una autonomía responsable, dentro de un trabajo colaborativo, multidisciplinar, en equipos, capaz de resolver problemas complejos y con capacidad de comunicar, transmitir y defender sus resultados.

- Determinar los procesos de coordinación que permitan el desarrollo en paralelo de las diferentes materias del mismo curso, y del resto de la titulación, y que favorezcan el apoyo mutuo del conocimiento a adquirir.

- Dotar a la metodología de un carácter flexible, de modo que permita ser aplicada en diferentes niveles, en función de la titulación, en el caso de otras ramas de conocimiento.

\section{Desarrollo de la innovación. Un cambio de concepto con los planteamientos clásicos de los PFC.}

EI TFG es un buen momento para poner en práctica la creación una dinámica de aprendizaje continua por parte del alumno. Es decir, iniciar a los alumnos en el autoaprendizaje, y como autogestionarlo, introduciéndoles en el cómo tendrán que hacerlo en su futuro profesional, en una sociedad más cambiante que nunca y que plantea nuevas necesidades.

Se plantea aquí, una propuesta para el desarrollo de los TFG, que modifica sustancialmente la forma de desarrollo más habitual, con la finalidad de realizar un ajuste más real de los trabajos a la finalidad de este tipo de materia, y permitir al estudiantado el desarrollo de su formación dentro de unos plazos concretos de tiempo (un curso).

Se plantea el TFG configurado como una asignatura colectiva de temporalidad anual, lo que da mayor margen para el "reposo" y reflexión para la fase de ideas, proceso de investigación, búsqueda de datos, toma de decisiones, con menor dedicación temporal semanal que si fuera 
cuatrimestral (al ser las mismas horas totales). De este modo se acota el plazo de ejecución, y el estudiante aprende al manejo de plazos parciales y totales.

El TFG debe tener una componente de realidad y relacionado con el ámbito de trabajo profesional, siendo capaz de desarrollar sus diferentes partes. Con un carácter eminentemente práctico, sin mayores aportaciones de conocimiento, de modo que permita interrelacionar los conocimientos adquiridos, acercando el mundo profesional.

El modo de trabajar es en conjunto. Con una distribución docente en grupos de 12 a 35 estudiantes, donde cada grupo desarrolla una misma temática establecida previamente por los tutores. Los trabajos dispondrán de dos fases fundamentales, una con carácter colectivo de inmersión en investigación en la temática objeto del TFG, y que servirá de base común para todo el alumnado, y su desarrollo posterior de propuestas, en equipos de 3 estudiantes, pero dentro del conjunto del grupo. Esto permite conocer el trabajo colectivo, la necesidad de los roles, la organización y responsabilidad de tareas. Se llega a profundizar en los temas hasta un nivel superior que el trabajo individual. Todo ello con dos o tres tutores por cada grupo, lo que permite al estudiante disponer de diferentes puntos de vista, lo que enriquece y obliga a generar una opinión propia.

Con el planteamiento de este nuevo modelo se plantean las siguientes prioridades:

- El contenido del trabajo y su desarrollo debe tener un carácter polivalente, su cronología debe ser clara y transparente para el estudiante y conocida de forma previa. Para ello se establece un dossier inicial de curso elaborado por los tutores.

- $\quad$ El TFG no debe ser solo un compendio de las habilidades y materias de la titulación. No se trata de aporta mayor nivel de conocimiento, sino de permitir demostrar el desarrollo simultáneo de las habilidades adquiridas durante el Grado.

- Se plantea complementar además otras competencias y habilidades, de carácter transversal (trabajo en equipo, capacidad de mostrar y defender el trabajo), que tendrán gran valor posterior en su desarrollo laboral.

- De este modo se cumplen, además de los objetivos fundamentales "oficiales" del TFG, respecto de mostrar las capacidades y habilidades adquiridas por el estudiante, aplicadas a la resolución de cuestiones reales y próximas a su desarrollo laboral, y dotar al estudiante de otras habilidades fundamentales en el mundo laboral actual.

- Podríamos considerarlo como el último paso del conocido estudio por competencias (proyecto Tuning), definido como la capacidad de movilizar recursos cognitivos para hacer frente a un tipo de situaciones (Perrenoud, 2004).

- Otro concepto del cambio propuesto es el de la presencialidad del profesorado. En el modelo propuesto al tratarse de la consideración de ser una materia, esta tiene implementada un número de horas de presencialidad por grupo, en la que además de tener incluidas la correspondiente al desarrollo de la materia se incluye la asistencia a los Tribunales. 


\subsection{El desarrollo de la propuesta en un curso}

Como se ha indicado se plantea que el TFG se desarrolle en un curso académico, con el siguiente tipo de actividades:

\subsubsection{Sesión Inicial}

Se establece con la totalidad de los estudiantes matriculados (asistencia obligatoria), y con representación de los tutores. Con las siguientes acciones:

Presentación del modelo de Trabajo, las temáticas previstas, los contenidos, finalidad y metodología a desarrollar, cronograma de acciones, y el proceso de evaluación.

Distribución de los estudiantes por grupos (caso de haber varias temáticas, según el número de estudiantes). En caso de haber una solicitud de estudiantes para un tema concreto mayor en número que las plazas disponibles, se establece un criterio de selección en función de la calificación obtenida en la materia afín a la temática elegida, o bien mejor expediente académico general.

Los estudiantes que desarrollan su TFG en estancias en el extranjero, o en otros Centros de Investigación o Universidades, se rigen por los mismos aspectos anteriores, si bien la presencialidad y seguimiento de su trabajo será especial.

\subsubsection{Sesiones de Grupo completo de estudiantes}

Una vez establecidos los grupos, los tutores establecen el método de desarrollo de la temática propuesta. A su vez se distribuyen los subtemas a desarrollar por cada equipo, así como los objetivos de cada uno de ellos. Se establecen las bases documentales y trabajos previos a realizar.

En una fase inicial se desarrolla una labor de investigación para tener un marco de referencia completo que permita un mejor conocimiento del tema al grupo y tener criterios y argumentos justificativos para la toma de decisiones en las fases siguientes.

Se planifica la asistencia periódica a las actividades y sesiones que se programen, y que tendrán su reflejo en el calendario del curso. Estas sesiones son de tres tipos:

Sesiones de Control o tutorización global, donde se analiza la evolución de cada trabajo y se establecen las premisas que con carácter general se aplican para todos los trabajos, así como las relaciones que deben existir de forma transversal entre todo el grupo. Se establece una periodicidad mínima de una al mes.

Sesiones de tutorización individual. Estas sesiones se dedican a la resolución de dudas por cada grupo de trabajo o estudiante. Dentro de las actividades de tutorización individual del profesor.

Sesiones de conferencia, visitas técnicas o charlas específicas de temas afines con el desarrollo del tema, a modo de seminarios. Se establecen de forma alterna con las sesiones de control. 
En la última sesión de control los tutores deben determinan si el trabajo desarrollado en el curso por el estudiante (o grupo) ha alcanzado el nivel suficiente, mediante una exposición a modo de "ensayo general" de la Defensa y la entrega de un dossier escrito. Si es superada se obtiene la autorización para ser presentado a su evaluación mediante Tribunal en sesión de Defensa Pública. Este paso implica la obtención del nivel mínimo de aprobado. En la fase siguiente se determina la calificación por encima de este valor. Se indican además las condiciones individuales para su presentación, recordando que es condición imprescindible tener aprobado el resto de los créditos de la titulación para proceder a la defensa del TFG. Caso de no ser así por parte de algún miembro de algún equipo se procede del modo más oportuno.

\subsubsection{La defensa pública ante Tribunal}

Una vez los estudiantes han obtenido el visto bueno de los tutores, la evaluación final se realiza ante Tribunal en sesión de Defensa Pública, con la entrega del dossier final definitivo, su exposición y defensa.

Cada Tribunal está formado por tres miembros, entre los profesores de la titulación que no han participado en el desarrollo del trabajo, y en caso de no haber quórum se incorporará al menos uno de los profesores tutores. No todos los trabajos tienen que ser evaluados por el mismo Tribunal.

Cada grupo debe entregar previo a su defensa, en formato digital, la presentación que se vaya a realizar ante el tribunal. Tiene un tiempo máximo de 30 minutos para presentar su defensa, y posteriormente estará a disposición del Tribunal para responder a las preguntas que se le hagan por otros 30 minutos.

Estas sesiones son públicas y abiertas a toda persona que quiera asistir, permitiendo el acceso a la sala, en los momentos previos a cada defensa, por respeto a los estudiantes que defienden su trabajo.

Finalizadas todas las sesiones de Tribunal, se reúne de forma conjunta la Comisión del TFG, formada por los Profesores que han conformado los Tribunales y los profesores tutores, junto con la Dirección del Centro, para proceder a la evaluación y establecimiento de calificaciones individuales de cada estudiante, teniendo en cuenta el proceso del Tribunal, junto con el desarrollo continuo del curso aportado por los tutores.

\subsection{La experiencia. TFG desde 2011-2012 a 2020-2021}

Para aprovechar la situación del grado de Ciencia y Tecnología de la Edificación, de ser el primer grado de la UAH en implantar el $4^{\circ}$ curso, y el primero en tener que implantar el nuevo modelo del Trabajo Final de Grado (TFG), con una carga de 12 ECTS, y un desarrollo previsto inicialmente en el segundo cuatrimestre de $4^{\circ}$ curso, se estableció el Proyecto de Innovación docente de la Universidad de Alcalá, con código UAH/EV358, que conllevaba una experiencia piloto (da Casa, 2012), iniciada el curso 2010/2011. En dicho curso no hubo estudiantes de TFG, pero se establecieron las bases, y planteamiento metodológico, sometido a debate en la comunidad universitaria de la Escuela. 
Desde el curso 2011/12 se ha mantenido el control experimental y la aplicación de mejoras continuadas, hasta la actualidad. Se debe indicar que se ha dejado fuera de este modelo lo correspondiente al Curso de Adaptación que se desarrolló en paralelo a la implantación de la titulación em los primeros años.

El número de estudiantes en estos cursos ha variado sustancialmente. Así en el curso 2011/12, hubo 32 estudiantes matriculados. En el curso 12/13, hubo 93 estudiantes. En el curso 13/14, hubo 79 estudiantes. Continuando el descenso progresivo hasya la actualidad, que en el curso 2020/21, han sido 25 los estudiantes matriculados.

Este descenso, está relacionado con la crisis económica de gran afección en el sector de la Construcción, y que ha repercutido en el interés por la titulación, si bien se debe indicar que se detecta una ligera recuperación en las matrículas de nuevo ingreso en nuestro grado.

\section{Resultados de la experiencia}

\subsection{Respecto de los contenidos desarrollados}

Los estudiantes consiguen durante el curso, una gran vinculación con la temática, a través de una primera fase de investigación y toma de datos, que permite conocer y poder disponer de criterios propios en la toma de decisiones posteriores, y por tanto el nivel de asimilación y desarrollo es muy alto, lo que se traduce en una gran implicación en todo el proceso. La posibilidad de realizar propuestas innovadoras, conociendo las consecuencias, consigue un resultado muy efectivo del trabajo, con una alta capacidad de defender sus argumentos por parte de los autores. El nivel alcanzado supera las expectativas iniciales de cada curso.

Es de significar que muchos estudiantes utilizan el dossier del TFG, a modo de ejemplarizar su capacidad de trabajo profesional, presentándolo en diferentes entrevistas de recursos humanos. Estos Departamentos nos han comunicado la gran visibilidad que para ellos tienen en dichos trabajos, los aspectos fundamentales que ellos buscan en sus procesos de selección.

Se presenta a continuación un breve resumen de los trabajos realizados en la experiencia en estos cursos:

4.1.1. Curso 11/12. Tema: La ciudad como laboratorio de la escuela. Estudio para la implantación de un campus universitario en Guadalajara

Se realiza un estudio del estado actual del funcionamiento de la ciudad de Guadalajara, para un análisis de la implantación de un campus universitario, en la ubicación que se determinara más adecuada. Se analizan los condicionantes y cómo influyen en la ciudad. Se analizan los factores de influencia de la nueva implantación y las consecuencias directas e indirectas, así como la modificación del funcionamiento actual de la ciudad en caso de implantación. Se analizan las posibles ubicaciones en la ciudad y tras un análisis comparativo se opta por una ubicación. Se determinan las necesidades del nuevo campus y se analizan las alternativas 
de organización, de acciones y su cronograma (fig 1). Todo ello se completa con el estudio económico de la implantación.

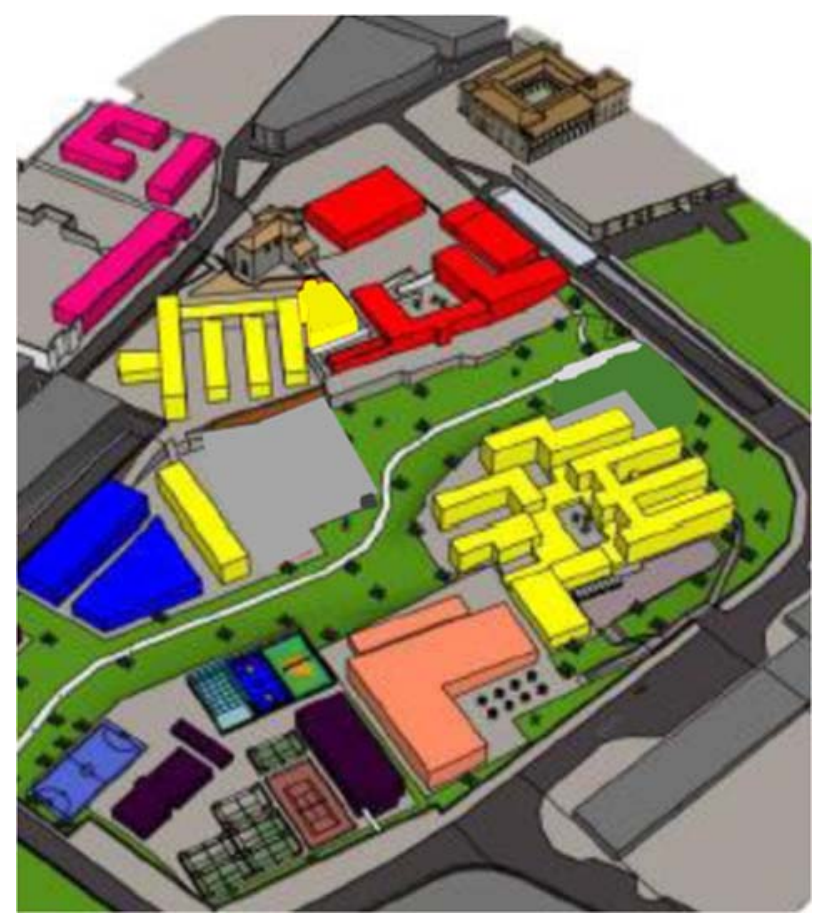

Fuente: Elaboración propia del curso

Fig. 1 Representación 3D del análisis de una de las propuestas. Fuente: Elaboración propia del curso.

\subsubsection{Curso 13/14. Tema: La gestión del Patrimonio Mundial. Aplicación en la Universidad de Alcalá}

El trabajo plantea realizar una aproximación interdisciplinar a diferentes modelos y fórmulas de planificación de la gestión del patrimonio histórico mundial. La Gestión es una herramienta fundamental, y más en el caso del Patrimonio Mundial. Se trata de integrar en un único proceso todos los factores, aspectos, o planes que suelen disponerse de forma individualizada para tener una visión de conjunto, coordinada y práctica, detectando y resolviendo las carencias y contradicciones, y aportando nuevas ideas para su desarrollo, optimización y puesta en valor (fig 2). El papel del técnico-gestor es fundamental.

El objetivo es enfocar todo el planteamiento al caso concreto de la Universidad de Alcalá, como parte del Patrimonio Mundial desde 1998. 
Se da una visión del tema enfocada al papel de la gestión como parte de la labor profesional en edificación, cada grupo de estudiantes plantea una propuesta concreta para poner en valor el Patrimonio de Alcalá en relación con la Universidad
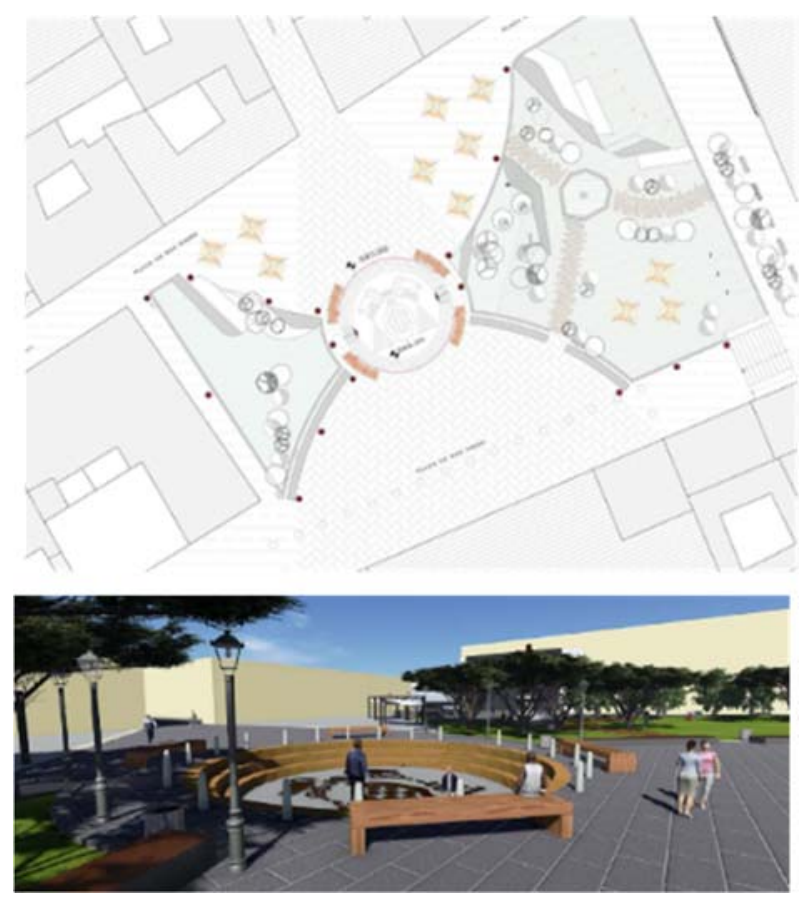

Fuente: Elaboración propia del curso

Fig. 2 Representación en planta y en 3D del análisis de una de las propuestas.

\subsubsection{Curso 15/16. Tema: La innovación aplicada a la edificación. Intervención en edificios} para su adecuación a un nuevo campus universitario del siglo XXI en Guadalajara”

La creación de un campus universitario acorde al siglo XXI, suponía integrar dicho campus con el entorno de la ciudad y adaptarlo a las necesidades que éste requería, respetando en todo momento el impacto que podría tener en la zona de intervención, considerando el análisis minucioso de la situación, junto con la fusión de la eficiencia energética y las energías renovables, para así poder alcanzar una sustentabilidad sólida e importante. Es una evolución del trabajo realizado en el curso 11/12, que se toma como referencia de partida.

Los aspectos de organización del campus, contribuyen a fomentar la participación e interrelación social con personas de diferentes formaciones académicas y experiencias profesionales, consiguiendo así una ampliación de perspectiva de trabajos y análisis, los cuales se deberán aplicar posteriormente en la vida laboral, en la cual se trabajará en equipos multidisciplinares (fig 3). 


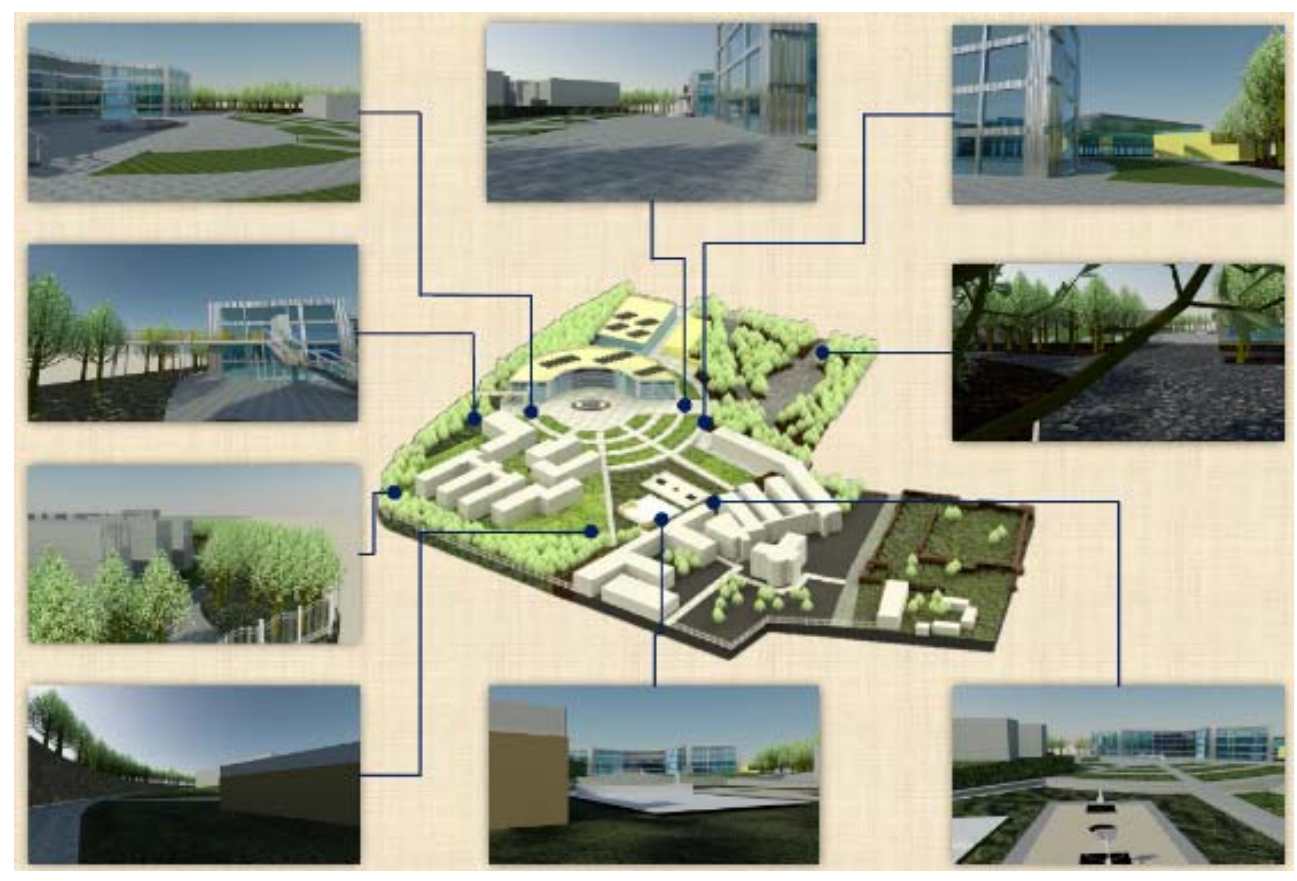

Fuente: Elaboración propia del curso

Fig. 3 Representación en 3D del análisis de una de las propuestas.

\subsubsection{Curso 19/20. Tema: La puesta en valor de la innovación tecnológica aplicada a la edificación}

Se presenta una gran oportunidad de aprovechar las nuevas tendencias del mercado, así como del campo de investigación sobre avances tecnológicos en la edificación y la obra civil. Algunas de las líneas en las tecnologías que están apareciendo ya en las edificaciones y procesos de construcción que nos rodean. La incorporación de procesos tecnológicos innovadores al sector de la edificación se ha dado gracias a la amplia utilidad y adaptación de estos.

Es a partir de esa premisa, donde se plantea como oportunidad la integración de esta temática como motivo principal del ejercicio académico del TFG, para tratarlo desde un enfoque especial, específico, técnico y cultural. La necesidad de integrar aspectos de nuevas tecnologías, sostenibilidad, usos, formas de entender, de vivir o disfrutar, y la implementación de nuevos elementos compatibles con la disposición de elementos construidos con otras 
técnicas y materiales (fig 4), es un reto para el que no hay una consideración profesional desarrollada.

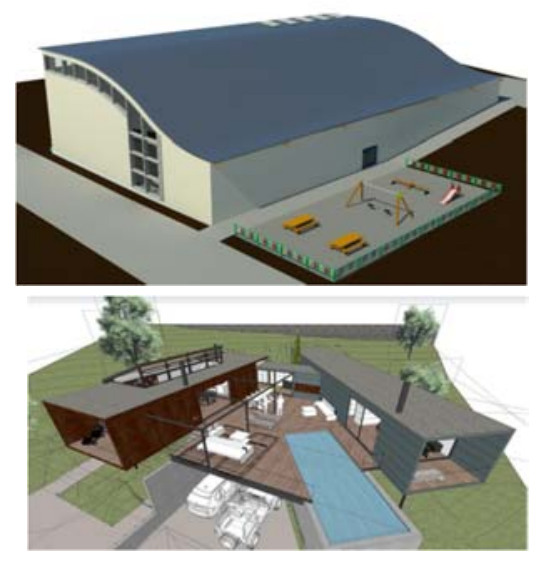

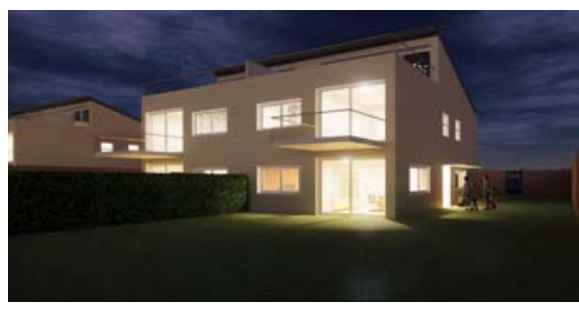

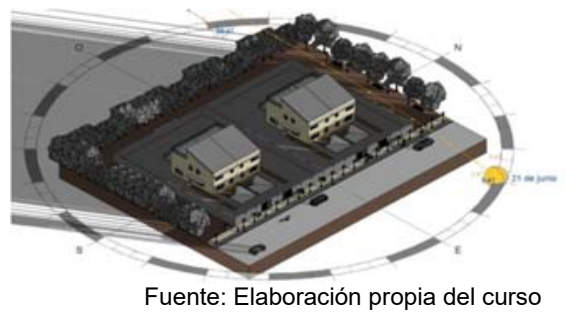

Fig. 4 Representación en 3D del análisis de varias de las propuestas. Fuente: Elaboración propia del curso.

\section{2. .- Respecto del estudiantado: participación y resultado}

La participación y seguimiento del estudiantado en los diferentes cursos ha sido muy alta, teniendo en cuenta el carácter presencial de las sesiones así establecidas. Plantear el curso de forma anual, y con el trabajo planificado de tal modo, permite que los estudiantes con materias pendientes puedan simultanearlo con el TFG y la coordinación con el profesorado, sin un gran esfuerzo adicional. El trabajo en equipo también favorece esta circunstancia.

El grado de eficiencia del resultado de los estudiantes del curso convencional es muy alto (no se consideran los datos del curso de adaptación por su situación de singularidad y no estar integrados en el modelo de estudio, ya que distorsionarían los resultados obtenidos del modelo propuesto).

Los estudiantes que desarrollan el curso, prácticamente la totalidad de los matriculados, superan el curso (92.9\%), en la totalidad de los cinco cursos solamente se ha suspendido a dos estudiantes (por no cumplir los requisitos de contenido en la documentación presentada al Tribunal). El dato del $6.5 \%$ de estudiantes que no presentaron sus trabajos es debido al no haber superado la totalidad de las materias de la titulación, por lo que no podían proceder a la Defensa ante Tribunal por norma.

Un aspecto relevante para conocer el nivel adquirido por los estudiantes es su propia opinión. Para ello podemos considerar las referencias que se obtienen de los trabajos finales, en sus valoraciones personales aportadas, que expresan de un modo claro el sentir al respecto. Por no ser extensivo, incorporamos algunas de estas referencias del pasado curso15/16, que resumen de un modo conciso este aspecto: 
La realización de este trabajo nos ha permitido desarrollar diferentes cualidades de trabajo en equipo, las cuales son muy importantes de cara a nuestro futuro personal y laboral.

La constante comunicación que ha existido en el desarrollo de la propuesta, ha facilitado el desarrollo de un espíritu de trabajo en equipo muy amplio, compartiendo una diversidad de opiniones, conocimientos y aptitudes.

La fluidez de esta comunicación y el apoyo constante entre los miembros del equipo, ha sido determinante para incentivar la motivación a lo largo del desarrollo del trabajo.

La motivación que se ha logrado desarrollar, ha sido la clave fundamental para alcanzar la perseverancia necesaria que requería la realización de esta propuesta, mejorando nuestra capacidad de respetar los plazos de entrega.

El conjunto de todas estas determinaciones, han sido la esencia fundamental del trabajo, las cuales nos han permitido avanzar en una misma dirección alcanzando los objetivos que nos habíamos propuesto. (García, 2016, p 98)

Desde otro punto de vista, se puede observar el nivel que los estudiantes muestran en su defensa ante el Tribunal, donde se llega a escuchar comentarios públicos en el debate por los miembros del Tribunal como "haber escuchado a un Grupo de profesionales".

\subsection{Respecto del profesorado participante}

Relevante es el interés mostrado por el profesorado. Su nivel de participación en el proceso evaluador ha sido muy alto. La participación es voluntaria, previa solicitud general, y siempre hay más candidatos que plazas, de hecho, en ocasiones se han configurado tribunales con más miembros de los tres requeridos. Se advierte la "ilusión" y motivación del profesorado, toda vez que la gran mayoría han sido estudiantes de sus materias y les permite ver la evolución de los mismos. Algunos profesores, han asistido a la sesión de Defensa, como público, al efecto de ver los resultados obtenidos. Es de agradecer dicha asistencia al mostrar el interés despertado por el modelo de trabajo.

Por otro lado, respecto del trabajo correspondiente a los tutores, el cambio del sistema obliga a una alta dedicación, si bien al tratarlo como una asignatura en equipo, y con la participación de varios profesores, este es más equilibrado. El cómputo de "carga docente" acordado con el Vicerrectorado, de $75 \mathrm{~h}$ por grupo (se ajusta a la docencia a impartir, e incluso incorpora las horas de Tribunal correspondientes. Este efecto se refuerza con el carácter voluntarista que caracteriza la Escuela, si bien el procedimiento descrito en este documento implica que queda poco margen para la "improvisación", o para la tutorización extemporánea y no organizada, más propia del proceso de tutorización del modelo anterior.

Un factor fundamental es que el profesorado participante dentro del proceso de esta materia, en su papel como "tutor o Director del trabajo" tenga un alto grado de coordinación, además de tener un gran compromiso con el proceso y metodología de trabajo. 


\subsection{Respecto del proceso evaluador: el Tribunal}

Los procesos de evaluación realizados hasta la fecha, han levantado un alto grado de expectación tanto entre el profesorado como en el alumnado, lo que se comprueba en la gran afluencia de público en las sesiones, además de familiares y amigos. Incluso se debe indicar que algunos profesores han manifestado su interés en participar en otras sesiones del mismo tipo de ejercicio de forma voluntaria.

Se debe indicar el alto grado de "efectividad" y "seriedad" que aporta al procedimiento el hecho que el Tribunal esté conformado por miembros del profesorado de la Escuela, pero que no formen parte del grupo de tutores, ya que de este modo los estudiantes consideran dicha evaluación, de mayor interés y con un grado de "neutralidad" ante el trabajo a presentar mucho mayor, favoreciendo el carácter profesional de la Defensa Pública del Trabajo, lo cual con posterioridad nos manifiestan los estudiantes que es de agradecer en su proceso de aprendizaje.

Los debates posteriores a la exposición tratan aspectos de gran profundidad, permitiendo a los alumnos desarrollar sus habilidades, consiguiendo transmitir su implicación y profesionalidad más allá de la exposición, aportando esto un aspecto diferencial muy positivo.

En todos los cursos se ha invitado a miembros del equipo rectoral a participar en alguna de las sesiones del Tribunal. Han asistido el Vicerrector del Campus de Guadalajara, el de Profesorado, el de Estudiantes, el Gerente de la Universidad, y la Directora de la Escuela de Arquitectura (antes de la fusión de centros), algunos de ellos han repetido. En el procedimiento de evaluación se ha visto con un alto grado de carácter positivo este hecho, con aporte de puntos de vista externos a la titulación.

\subsection{Respecto de la trascendencia del modelo de proceso para el TFG}

El modelo de TFG, en su fase inicial, fue propuesto para su desarrollo y aplicación de forma oficial en la Titulación de Ciencia y Tecnología de la Edificación, siendo aprobado en Junta de Centro de 13/12/2010. El resultado del Proyecto de innovación docente referido, en su informe recopilatorio del curso 2013/2014, (da Casa, 2014) fue utilizado como base para la redacción de la Normativa de Trabajos Fin de Grado de la UAH, aprobada en Consejo de Gobierno el 18/06/2015 (UAH 2016).

El Reglamento específico para el Grado de Ciencia y Tecnología de la Edificación, de la Escuela de Arquitectura, se modificó y fue aprobado en Junta de Escuela de Arquitectura de 19/10/2015, e informada favorablemente en Comisión de Docencia de la UAH el 4/12/2015. Siendo de aplicación desde el curso 2015/2016. Desde entonces se ha continuado con la implantación progresiva de los resultados del proceso de mejora, tras el análisis de los resultados de cada año, y el planteamiento del curso siguiente. 


\section{Conclusiones}

Se podría concluir que el TFG se debe considerar como el primer paso del aprendizaje continuado o aprendizaje permanente (Long Life Learning) como parte de la filosofía que fundamenta el EEES y que permite hacer que las personas puedan adaptarse mejor a los cambios continuos que se muestran en la sociedad y por tanto en el mundo laboral y empresarial, aspecto que además ya aparecía en el eje $n^{\circ} 9$ de la "Estrategia Universidad 2015" (Ministerio de Educación 2011).

La propuesta de metodología presentada y experimentada como experiencia de forma continuada desde 2011, presenta un modelo experimentado y aplicado, en base a criterios participativos y activos por parte del alumno, y relacionado con la resolución de problemas con una fuerte componente real, y en base al trabajo multidisciplinar, en equipos, y con una fuerte necesidad de exponer y defender las soluciones propuestas, con un resultado óptimo, que se muestra en los indicadores relativos del alumnado, favoreciendo la continuidad de los alumnos del curso, y con ello de superación de las materias. Además, en su proceso final, el alumno obtiene una serie de competencias trasversales como son: autonomía responsable, trabajo colaborativo, capacidad de resolver problemas complejos, y con capacidad de comunicar, transmitir y defender sus resultados. La calidad y profundidad técnica de los trabajos deja patente la evolución del alumnado. Todo ello redunda en una aproximación al mundo laboral.

Además, el método planteado es fácilmente exportable a cualquier tipo de titulación, independientemente de la Rama de Conocimiento, y entra dentro de los habituales procesos de cómputo de la dedicación docente del profesorado. El proceso de control y mejora continua desarrollado, permite disponer de un modelo más avanzado, y con mayor garantía de resultado para no caer en las dificultades ya consideradas, pudiendo optar por la solución que más se adapte a las características propias de cada titulación.

\section{Referencias}

ANECA (2012) Guía de Apoyo para la elaboración de la memoria de verificación de títulos oficiales universitarios (Grado y Máster) Agencia Nacional de Evaluación de la Calidad y Acreditación (ANECA) Recuperado de https://www.uchceu.es/docs/calidad/tramite-titulaciones/guia-ANECA.pdf

ARISTIZABAL, J.L.; RAMOS, A.; CHIRINO, V. (2018). Aprendizaje activo para el desarrollo de la psicomotricidad y el trabajo en equipo. Revista Electrónica Educare (Educare Electronic Journal) Vol. 22(1)

DA CASA, F., GARCíA, A., BAÑO, A., RODRÍGUEZ, F.J., MARÍN, A., CORIA, G., ... , DELGADO, I. (2008) "Sistema de evaluación y gestión del TFC. La comisión del tribunal final de carrera de la EUAT de Guadalajara de la UAH“. Actas del I Encuentro Internacional Profesores Proyecto Final de Carrera de Arquitectura Técnica. Ed Universidad Politécnica de Valencia.

DA CASA, F., (2012). "EI Trabajo Final de Grado. Una experiencia innovadora aplicada en las titulaciones Técnicas". VI Encuentro de Innovación en Docencia Universitaria Propuestas interdisciplinares e integradas. Universidad de Alcalá. 2012. 
DA CASA, F., GARCÍA, A., FERNÁNDEZ, E. (2014) "La implantación del Trabajo Final de Grado en las nuevas titulaciones. La situación concreta del grado de Ingeniería de Edificación (ciencia y tecnología en la edificación) como experiencia piloto en la Universidad de Alcalá (proyecto de innovación docente UAH/EV358)". XIII International Conference on Engineering and Technology Education. Guimarães - PORTUGAL.

GARCÍA, C., GARCÍA, F., ORTIZ, S. (2016) "La innovación aplicada a la edificación. Intervención en un nuevo campus universitario del siglo XXI en Guadalajara. Adecuación y multifuncionalidad del campus, sostenibilidad energética y gestión de la energía eléctrica" Trabajo de Fin de Grado Ciencia y Tecnología de la Edificación, Curso 2015/2016. Universidad de Alcalá.

MINISTERIO DE EDUCACIÓN (2011) Estrategia universidad 2015. Contribución de las universidades al progreso socioeconómico español 2010-2015. Octubre 2010. Secretaría General de Universidades. Ministerio de Educación. Madrid

OJEDA, J. (2019). Técnicas activas y su contribución al aprendizaje de la matemática en estudiantes de séptimo grado. CIENCIAMATRIA, 5(9), 517-535. https://doi.org/10.35381/cm.v5i9.211

PERRENOUD, P. (2004). Diez nuevas competencias para ensenar. Editorial Grao. Barcelona (España).

RULLÁN, M; FERNÁNDEZ, M, ESTAPE, G, MÁRQUEZ, M.D. (2010). "La evaluación de competencias transversales en la materia trabajos de fin de grado. Un estudio preliminar sobre la necesidad y oportunidad de establecer medios e instrumentos por ramas de conocimiento". Revista de Docencia Universitaria, Vol. 1. № 8: 74า100.

UAH (2016) Normativa sobre los Trabajos Fin de Grado de la Universidad de Alcalá. Recuperado de https://www.uah.es/export/sites/uah/es/conoce-la-uah/organizacion-y-gobierno/.galleries/GaleriaSecretaria-General/Normativa-Trabajos-Fin-Grado.pdf

UCM (2016) Instrucciones para elaboración de la Tesina. Facultad de Biología. Universidad Complutense de Madrid. Recuperado dehttp://biologicas.ucm.es/tesina_de_licenciatura_1

UNAV (2016) Instrucciones para elaboración de la Tesina. Facultad de Teología. UNAV. Recuperado de www.unav.edu/web 